\title{
Design, Analysis, and Control of a Stiffness-Tunable 3-DOF Rubber-Bearing Positioning Stage
}

\author{
Chi-An Chen ${ }^{1 a^{*}}$, Yu-Cheng Chen ${ }^{1 a}$, Kuo-Shen Chen ${ }^{1 b}$, Yun-Hui Liu ${ }^{2 b}$ \\ ${ }^{\mathrm{a}}$ Graduate Student, ${ }^{\mathrm{b}}$ Professor \\ ${ }^{1}$ Department of Mechanical Engineering, National Cheng-Kung University, \\ No.1, Daxue Rd., East Dist., Tainan City 701, Taiwan \\ ${ }^{2}$ Department of Mechanical Engineering, Southern Taiwan University of Science and Technology, \\ No. 1, Nantai St., Yungkang Dist., Tainan 71005, Taiwan \\ *Corresponding Author: a850405tw@outlook.com
}

\begin{abstract}
A 3-DOF $\theta_{x}-Y-\theta_{z}$ rubber bearing stage with stiffness-adjustment capability is design, realized, and controlled in this work. The stiffness of rubbers can be adjusted easily by adding a preload from different directions. Base on elastomeric materials analysis, the stiffness of rubber and therefore dynamic characteristics of the stage can be changed with such a preload. In comparison with positioning stages designed by compliance mechanism approach, such characteristics could be an opportunity to improve the performance by replacing compliant bearing with rubber-bearing one. The dynamic testing results show that with a compression of $0.8 \mathrm{~mm}$ on a $1.5 \mathrm{~mm}$ thick rubber, the stiffness and natural frequency increased by $100 \%$ and $30 \%$ in $Y$-axis, $15 \%$ and $7 \%$ in $\theta_{x}$-axis, and even by $510 \%$ and $140 \%$ in $\theta_{z}$-axis, respectively. Associated PID and integral sliding mode controllers are then implemented for characterizing the position control performance. Currently, the rubber bearing stage can achieve a $79 \mathrm{~Hz}$ and $110 \mathrm{~Hz}$ controlling bandwidth by using PID and integral sliding mode controller (ISMC), respectively in $Y$-axis motion. In the future, the effect of different pre-compression displacement on dynamics characteristics of the stage, such as stiffness, natural frequency, damping ratio, will be tested. Additional control design in multi-axis coupling will be addressed to evaluate the performance in precision motion related applications.
\end{abstract}

Keywords: elastomeric bearing, positioning stage, feedback control.

\section{Introduction}

Positioning stage have been widely used in manufacturing system and precision metrology ${ }^{(1)}$. For example, fast steering mirrors, which is used in laser manufacturing related applications, comprise rotational positioning stages ${ }^{(2)}$. Such stages required higher precision, high bandwidth, and high resolution for the purpose of enhancing manufacturing capability. Traditionally, these stages are usually designed and realized based on compliant mechanisms ${ }^{(3)}$. It provides stiffness by its compliant structure. For example, compliant stages usually utilize beam flexural deformation to gain the required compliance, which depends on the geometry and material properties. For example, for achieving a high compliant structure, the structural length could be long and is not adjustable once the structure is realized. If the dynamics characteristics of stage need to be adjusted, the shape of compliant structure would need to be re-design and this causes inconveniences.

Elastomeric bearings (aka rubber bearings) are essentially rubber-metallic laminates structures. They are used in building technologies to reduce vibration for many years $^{(4)}$. Recently, this kind of elastomeric materials have been integrated into precision stage design. By changing the shape factor of rubbers, the stiffness under compression can be much higher than that in shearing direction ${ }^{(5)}$. With proper design, the strong stiffness anisotropy can be exhibited that the motion in shear direction can be flexible while the motion in compression direction be blocked effectively. However, elastomeric bearings are viscoelastic 
material, its behavior depends on mechanical properties of materials, operating condition and geometry. The characteristics also influenced by temperature. It is important to perform essential characterization for fully taking the advantage of this approach.

For example, Kulk ${ }^{(6)}$ designed a fast steering mirror for optical communication. With elastomeric bearings, the system exhibited a stroke of 3.5-mrad angular motion with $10-\mathrm{kHz}$ bandwidth. Teng ${ }^{(7)}$ developed a one-dimensional elastomeric bearing positioning stage that provide a $139 \mu \mathrm{m}$ stroke. With PID controller, the stage can have a $27-\mathrm{Hz}$ bandwidth, the bandwidth is increased to 350 by using integral sliding mode controller (ISMC). In our previous work, a 3-DOF elastomeric bearing stage has also been developed $^{(8)}$, the bandwidths are $80 \mathrm{~Hz}, 62 \mathrm{~Hz}$ and $54 \mathrm{~Hz}$ in $Y$-, $\theta_{x}$-and- $\theta_{z}$ axis. In that work, it also showed that the shearing stiffness and natural frequency could be adjustable with adding preload in compression direction ${ }^{(8)}$. This could be an advantage for rubber bearing stage that the dynamic model can be tuned easily. Therefore, it is important to design a stage with adjustable preloading for evaluating the effect of preloading on the characteristic dynamic and control performance systematically for future related applications.

The motivation of the research is, therefore, to realize a novel 3-DOF stage which can provide pre-load on rubber bearing in order to make its dynamics characteristics adjustable easily. In this work, we apply viscoelastic dynamics to developed system models of stage. Meanwhile, two controllers have also been designed to realized positioning experiment. Notice that although our previous investigation ${ }^{(8)}$ dealt with all possible deformation types, this work only focuses on shearing stiffness only and it leaves as our future work for exploring the rubber dynamics subjected to other types of pre-loads.

The research flow is shown in Fig. 1. First is to establish the rubber bearing stage with preload-adjustment, followed by dynamic characterization for obtaining the system model. Furthermore, the dynamic experiment with adding preload is demoed to show how the stage model dynamics be adjustable in $Y$-, $\theta_{x}$-and- $\theta_{z}$ axis. Next, PID controller and integration sliding model controller (ISMC) has been designed and used for positioning experiment in $Y$-axis.

The rest of this article presents the work in detail. The conceptual design of the stage and realization are presented in Section 2. Next, system dynamic test is presented in Section 3. In Section 4, the design of the controller is presented and followed by the positioning experiment addressed in Section 5. Finally, Section 6 concludes the paper.

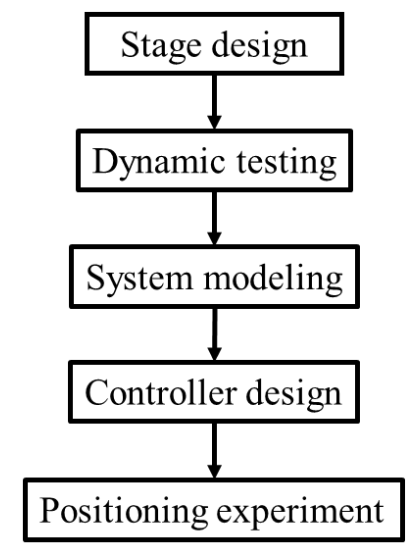

Fig. 1. Research flow of stage realization.

\section{Stage Design and Realization}

\subsection{3-DOF Positioning Stage}

A 3-DOF rubber bearing positioning stage has been developed $^{(8)}$. This stage consists of one translation ( $Y$-axis) and two rotational ( $\theta_{x}$ and $\theta_{z}$-axis) DOF, the Schematic plot for stage design is shown in Fig. 2. As shown in Fig. 3a, three AVM40-20 voice coil motors (VCM) are used to actuate the stage. Three ASP-50-CTA capacitive probes are the sensor to capture the stage motion. Four rubber pads $\left(15 \times 15 \times 1.5 \mathrm{~mm}^{3}\right)$ are attached on an aluminum block $\left(30 \times 30 \times 30 \mathrm{~mm}^{3}\right)$ as the main body of the stage. The stiffness of the rubber bearing stage $\left(k_{y}, k_{\theta x}\right.$ and $\left.k_{\theta z}\right)$ is express by shearing stiffness $\left(k_{s}\right)$, torsional stiffness $\left(k_{t}\right)$ and bending stiffness $\left(k_{b}\right)^{(8)}$. The stiffness of rubber bearing $k_{s}, k_{t}$ and $k_{b}$ are $120 \mathrm{~N} / \mathrm{mm}, 3.81 \mathrm{Nm} / \mathrm{rad}$ and $11.25 \mathrm{Nm} / \mathrm{rad}$ by testing method. The equation of motion of stage can be obtained as

$$
\begin{aligned}
& M_{y} \ddot{Y}+C_{y} \dot{Y}+k_{y} Y=F_{y} \\
& I_{x x} \ddot{\theta}_{x}+C_{x} \dot{\theta}_{x}+k_{\theta_{x}} \theta_{x}=M_{x} \\
& I_{z z} \ddot{\theta}_{z}+C_{z} \dot{\theta}_{z}+k_{\theta_{z}} \theta_{z}=M_{z}
\end{aligned}
$$

The coordinates of force and measurement displacement need to be transformed by 3 voice coil motors $\left(F_{1}, F_{2}\right.$ and $\left.F_{3}\right)$ and three capacitive sensors $\left(Y_{1}, Y_{2}\right.$ and $\left.Y_{3}\right)$. The relations show in Chen's thesis ${ }^{(8)}$, it related to the arm length of force $\left(a_{f}\right)$ and measurement point $\left(a_{y}\right)$. Rubber bearing of this positioning stage cannot be adding preload, 
which means the dynamics characteristics is un-adjustable. In contrast, a rubber-adjustment design has installed to the stage, as shown in Fig.3, four mechanisms are used to compress rubbers. This could make the dynamic characteristics of stage change.

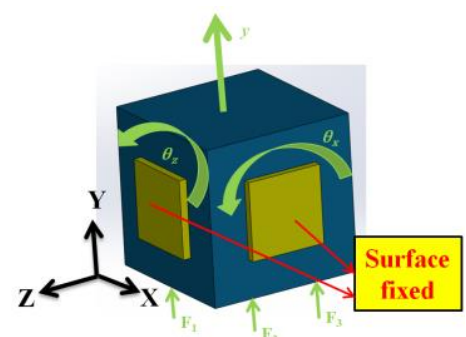

Fig. 2. Schematic plot for stage design.

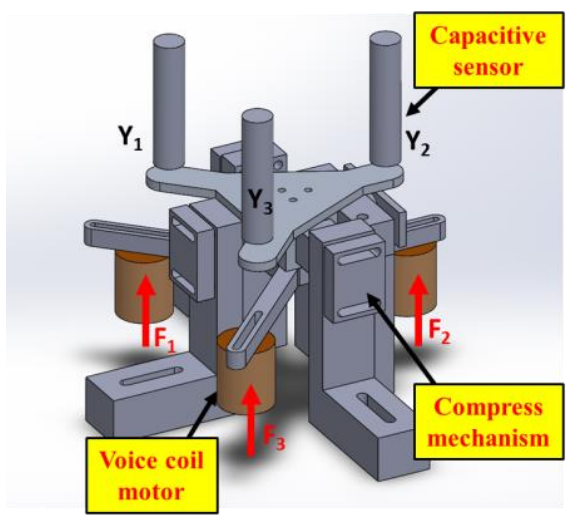

(a) schematic plot of stage.

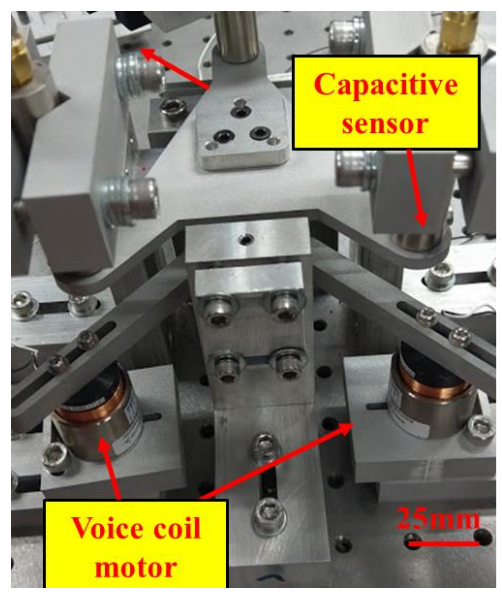

(b) stage realization.

Fig. 3. Rubber bearing stage.

\subsection{Rubber Stiffness Adjustment Design}

The rubber stiffness is characterized by a material testing system, called $\mathrm{HSC}^{(8)}$, which provides a compression loading in compress direction of rubber by step motor. It can test shear stiffness under different preload. The experiment data shows that with different preload, the shear stiffness increases furthermore, the natural frequency and damping ratio of rubber bearing also change with adding preload. With this result, we can see that the rubber can adjust easily with different loading condition.

A new 3-DOF rubber bearing stage has been developed, as Fig. 3 shown. Smaller VCMs AVM30-15 have been chosen to make the mass of stage lighter. The parameters of $a_{f}$ and $a_{y}$ are $62.5 \mathrm{~mm}$ and $50 \mathrm{~mm}$. Fig. 4 shows the design that rubbers can be compress and fixed by screws in four directions. Compare to HSC system, not only shear stiffness relates to system dynamics, the bending stiffness and torsional stiffness also need to be considered. The entire control flow is shown in Fig. 5.

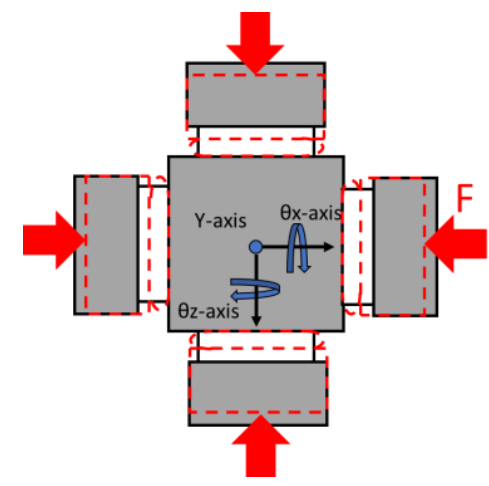

(a) preload adjustment design.

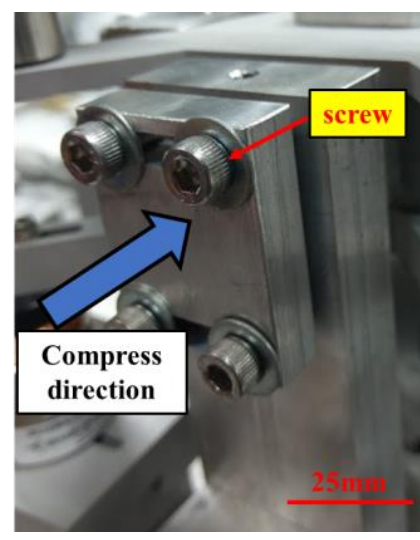

(b) preload adjustment realization.

Fig. 4. Rubber stiffness adjustment design.

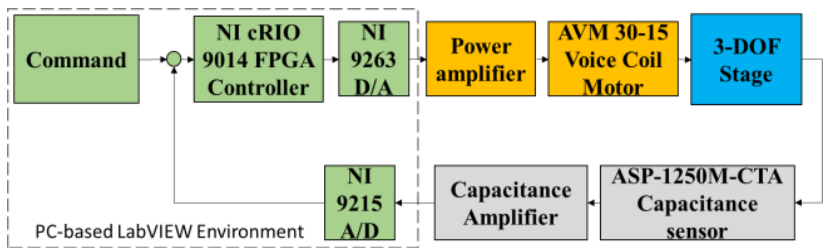

Fig. 5. Block diagram for controlling the system. 


\section{System Dynamic Test}

\subsection{System Dynamics Modeling}

The system model is established by step and sinusoidal response. Since rubbers are viscoelastic materials, thus a generalized Maxwell model is used to perform a time- and frequency-varying spring $K(s)$, which combined with three linear springs $\left(k_{1}, k_{2}\right.$ and $\left.k_{3}\right)$ and two dampers $\left(c_{2} \text { and } c_{3}\right)^{(9)}$. The stage model is shown as Fig. 6 . The system model could be expressed as

$$
G_{i j}=\frac{1}{M_{i j} s^{2}+C_{i j} s+K_{i j}(s)} \times G_{v}
$$

Here the time dependent stiffness, $K_{i j}$, can represent the creeping effect of rubber bearing stiffness ${ }^{(7)}$. $G_{v}$ denotes the first-order order system of voice coil motor, the cut-off frequency $\omega_{v}$ is $300 \times 2 \pi$, and $G_{i j}$ represent the transfer function between $i$-axis output and $j$-axis input with the units of $\mu \mathrm{m} / \mathrm{V}$ for translation axis and $\mathrm{mrad} / \mathrm{V}$ for rotation axis, respectively. The models of system are established by curve fitting. For example, the parameters of $G_{y y}$ is shown in Table 1. Comparison of the simulation and the experimental results are shown in Fig. 7. The creep effect of rubber makes the stiffness of stage decrease.

The coupled effect also established by fitting step response and sinusoidal response. The transfer function models of coupled axis are much more complicated. Fig. 8 and Fig. 9 shows the simulation and experimental result of $G_{x y}$ and $G_{z y}$.

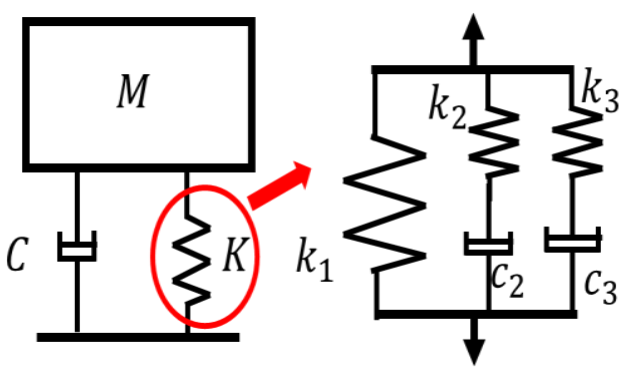

Fig. 6. Stage viscoelastic model.

Table 1. The parameters of $G_{y y}$.

\begin{tabular}{|c|c|c|c|}
\hline & $M$ & $C$ & $k_{1}$ \\
\cline { 2 - 4 } & $1.17 \times 10^{-5}$ & 0.0025 & 20.41 \\
\hline$k_{2}$ & $k_{3}$ & $c_{2}$ & $c_{3}$ \\
\hline 2.45 & 1.84 & 1.2245 & 0.1224 \\
\hline
\end{tabular}
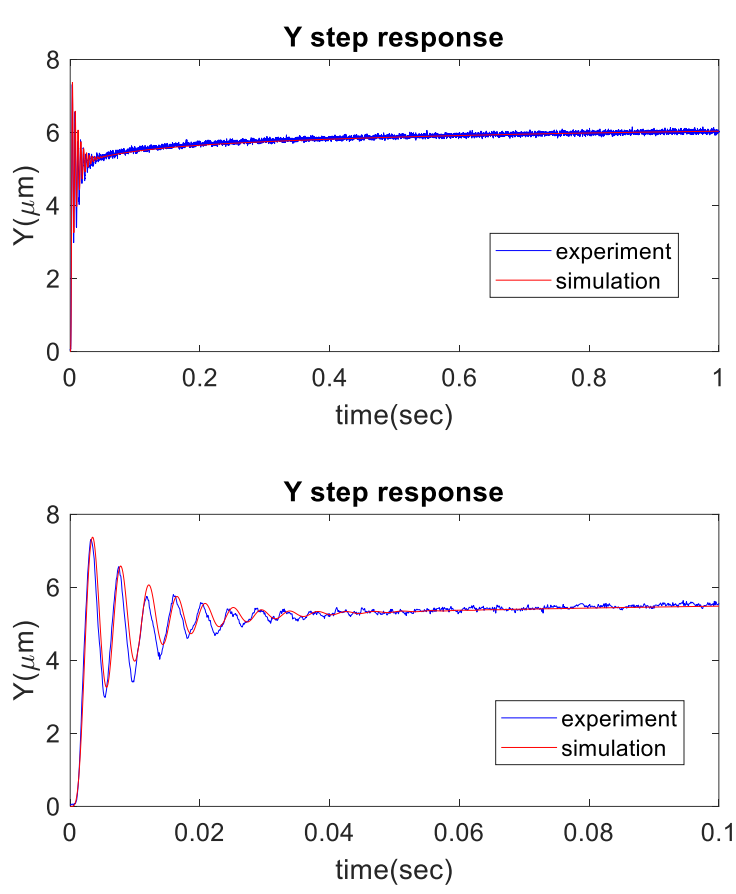

(a) $Y$-axis step response.
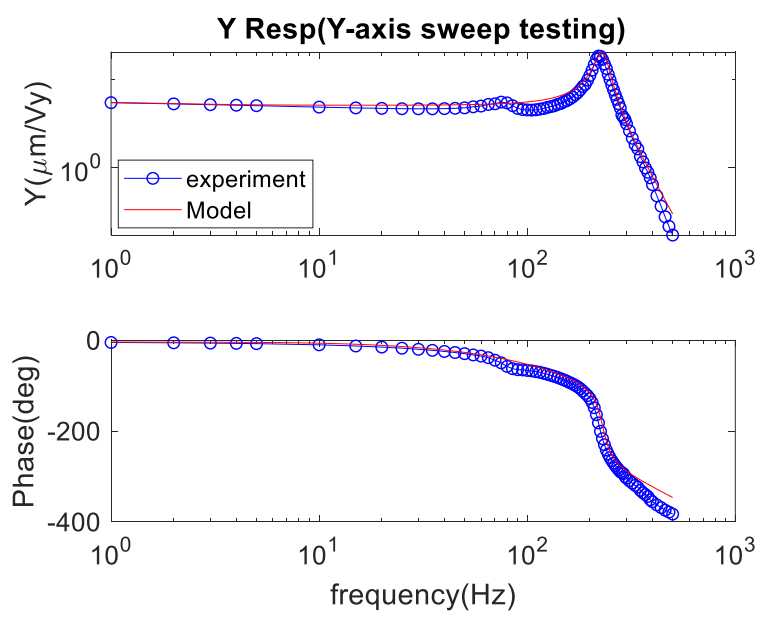

(b) bode diagram of $Y$-axis.

Fig. 7. Dynamics model of $Y$-axis.
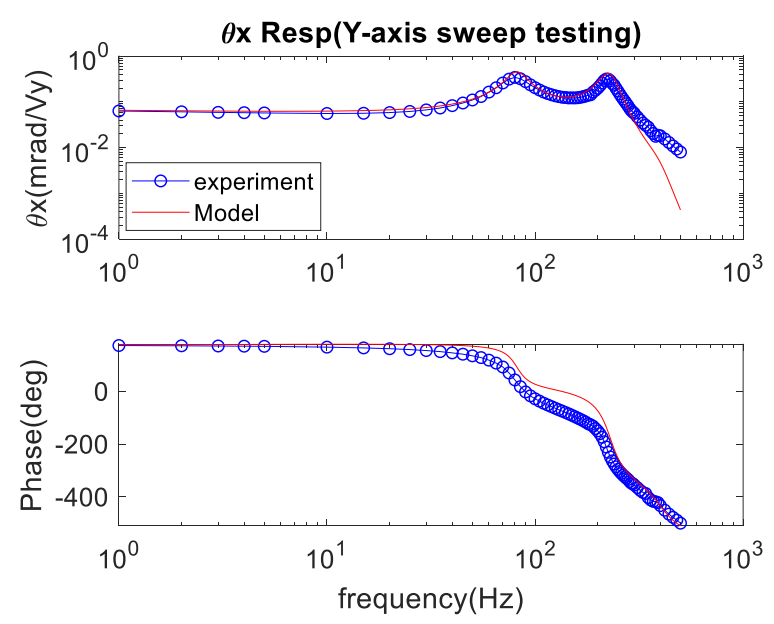

Fig. 8. Dynamics model of $\theta_{x}$-axis. 

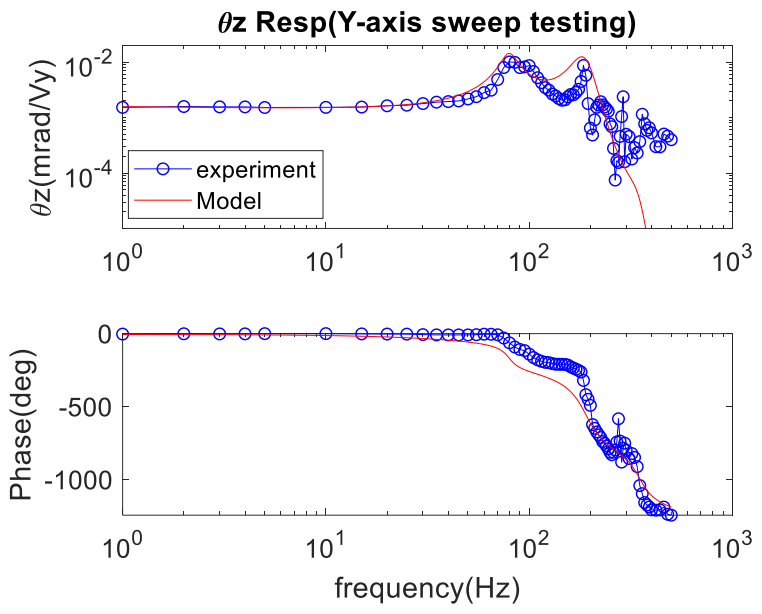

Fig. 9. Dynamics model of $\theta_{z}$-axis.

\subsection{Dynamic experiment with preload}

In this part we add preload to compress rubber bearing and show how the dynamic models are influenced. The rubbers have been adding preload and compressed $0.8 \mathrm{~mm}$ in $\theta_{x}$-direction as shown in Fig. 9. In this situation, compress $k_{s}$ makes $k_{y}$ change, $k_{\theta x}$ is affect by compress $k_{s}$ and $k_{t}$, and $k_{\theta z}$ is influenced by $k_{b}$ and $k_{s}$. By sinusoidal response test, transfer function of $G_{y y}, G_{x x}$ and $G_{z z}$ has change, the results are shown in Fig. 10. The stiffness and natural frequency increase with adding preload. Table 2 shows the comparison of dynamic characteristics. $G_{z z}$ has significant change in both stiffness and natural frequency, in the other hand, dynamic characteristic of $G_{x x}$ does not alter so much.

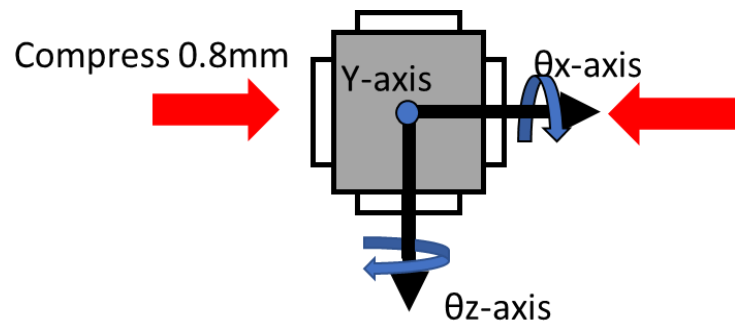

Fig. 9. Compress rubber bearing condition.

Table 2. Characteristics of rubber bearing stage.

\begin{tabular}{|c|c|c|c|c|}
\hline \multirow{2}{*}{} & \multicolumn{2}{|c|}{$\begin{array}{c}\text { Stiffness }\left(\mathrm{V}_{\mathrm{i}} / \mathrm{um}, \mathrm{V}_{\mathrm{i}} / \mathrm{mrad}\right) \\
(\mathrm{i}=\mathrm{y}, \mathrm{x}, \mathrm{z})\end{array}$} & \multicolumn{2}{c|}{ Natural frequency $(\mathrm{Hz})$} \\
\cline { 2 - 5 } & original & $\begin{array}{c}\text { Compress } \\
0.8 \mathrm{~mm}\end{array}$ & original & $\begin{array}{c}\text { Compress } \\
0.8 \mathrm{~mm}\end{array}$ \\
\hline$Y$ & 0.183 & 0.367 & 225 & 295 \\
\hline$\theta_{x}$ & 0.441 & 0.503 & 75 & 80 \\
\hline$\theta_{z}$ & 0.733 & 4.481 & 80 & 190 \\
\hline
\end{tabular}
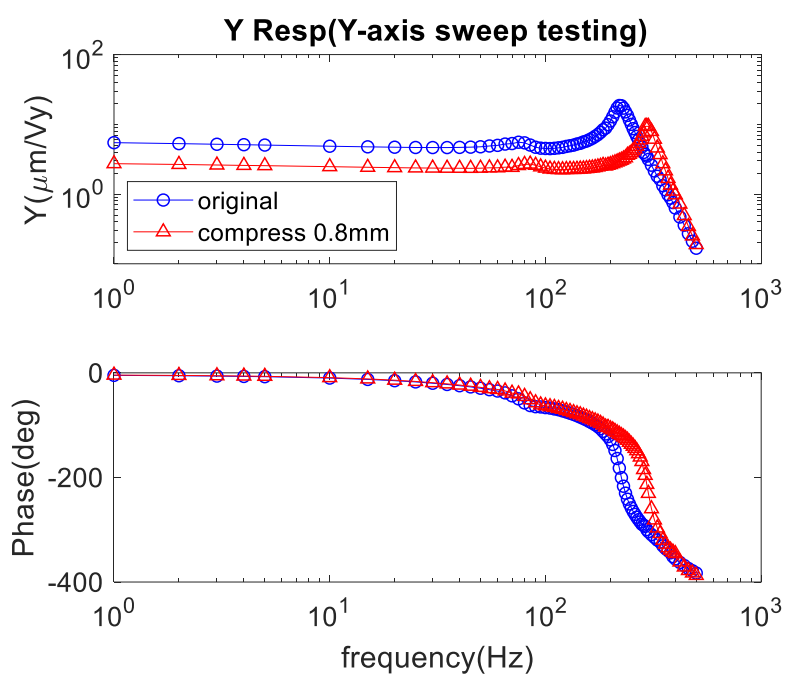

(a) $Y$-axis response
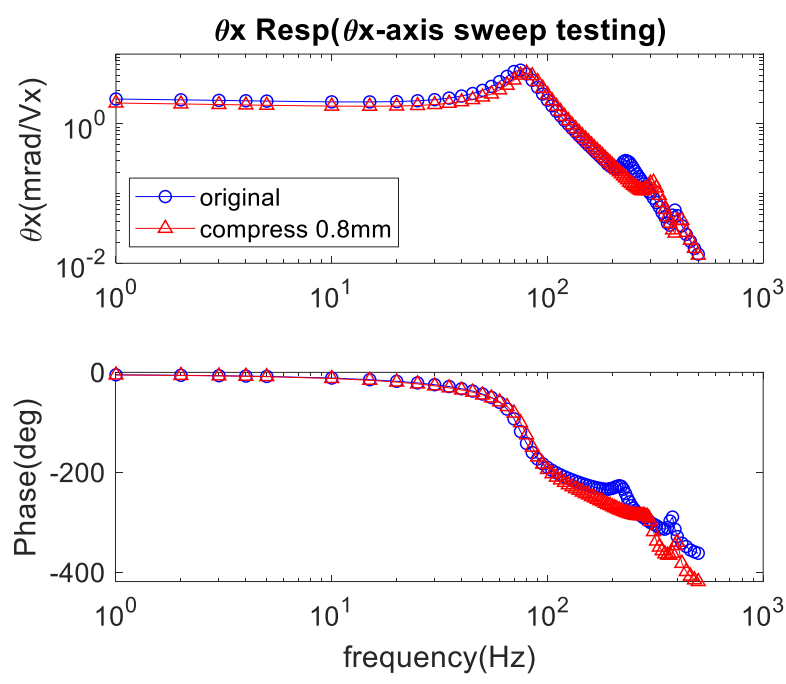

(b) $\theta x$-axis response.
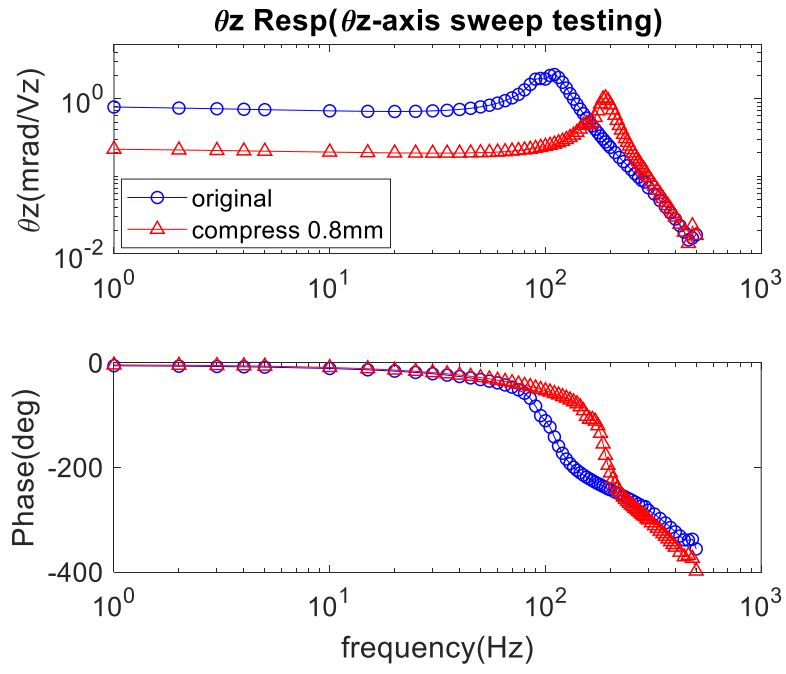

(c) $\theta z$-axis response.

Fig. 10. Bode diagram with adding preload. 


\section{Controller Design}

There are two controllers has used for stage control: PID controller and integral sliding mode control. PID control is selected to be a basic threshold of control performance. After PID controller established, integral sliding mode control will be applied to seek a better control result.

\subsection{PID control}

PID controller is widely used in control application ${ }^{(10)}$. With appropriate design, the resonance peak of bode diagram can be suppressed effectively. The traditional PID controller are written as

$$
G_{\text {pid }}=k_{p}\left(+\frac{1}{T_{i} S}+T_{d} s\right)
$$

Where $k_{p}$ is proportional gain, $T_{i}$ is integral gain and $T_{d}$ is differential gain.

\subsection{Integral sliding mode control}

On the other hand, the integral sliding mode control (ISMC) is also be designed ${ }^{(11)}$. A sliding function $s(x, t)$ has been chosen, which is defined as

$$
s(x, t)=\left(\frac{d}{d t}+\lambda\right)^{n-1} \tilde{x}+\beta \int_{0}^{t} \tilde{x}
$$

Where $\tilde{x}=x(t)-x_{d}(t) . x_{d}$ is the command of stage position. There are four parameters of ISMC: $\lambda, \eta, \phi$ and $\beta$. Please refer to Deng's work $^{(7)}$ for the detail in determining of ISMC's control parameters.

\section{Control Result}

The NI cRIO-9014 FPGA is chosen as the real time controlling platform for developing controller to conduct positioning experiment. The sampling rate $\left(F_{s}\right)$ of system is $10-\mathrm{kHz}$. A $15.7 \mu \mathrm{m}$ step input in $Y$-axis direction is command. Step response and sinusoidal response are tested.

\subsection{Comparison with different dynamic model}

There are 3 control result with PID controller shown in Fig. 11. First, we control the stage with PID gain $k_{p}=3$, $T_{i}=0.7 / F_{s}$ and $T_{d}=0.5 / F_{s}$ can reach a rise time and settling time of $6 \mathrm{~ms}$ and $25.3 \mathrm{~ms}$ with a $5.1 \%$ overshoot (PID-a).
Second, the rubbers were compressed $0.8 \mathrm{~mm}$ in $\theta_{x}$ -direction without adjust PID gain (PID-b). The result has different due to the plant change. The rise time and settling time are $11.8 \mathrm{~ms}$ and $41.4 \mathrm{~ms}$ in this condition. Final, another PID gain $\left(k_{p}=4.5, T_{i}=0.73 / F_{s}\right.$ and $\left.T_{d}=0.02 / F_{s}\right)$ has been chosen to make the performance better, it can achieve a rise time and settling time of $5.8 \mathrm{~ms}$ and $24.3 \mathrm{~ms}$ in current work. Fig. 12 shows the sinusoidal test result. The $-3 \mathrm{~dB}$ bandwidths are $79 \mathrm{~Hz}, 35 \mathrm{~Hz}$ and $79 \mathrm{~Hz}$ in these 3 conditions. From PID-a and PID-b, the bode plot of close loop system are different because the dynamic model has changed. It shows that PID-c can reach a same bandwidth as that of PID-a, due to the increase of natural frequency from $210 \mathrm{~Hz}$ to $250 \mathrm{~Hz}$ with preload.

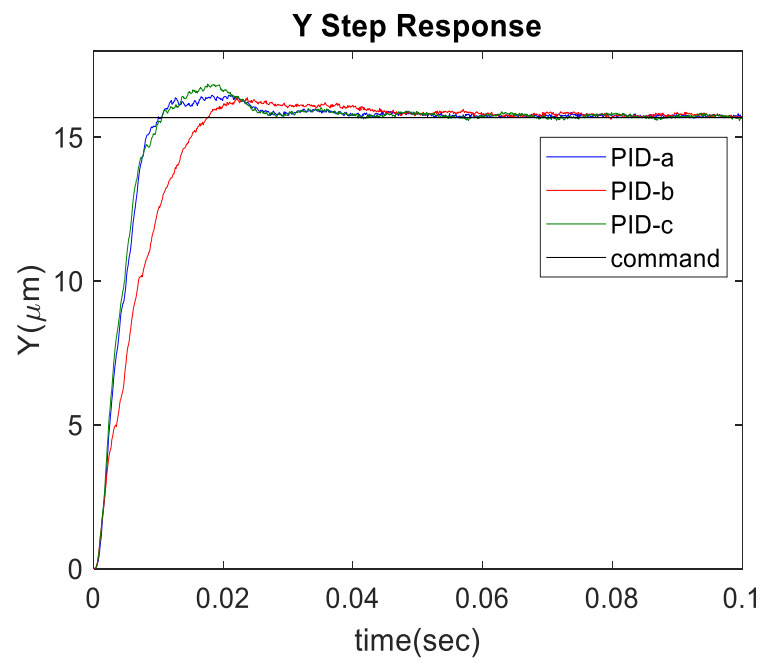

Fig. 11. Step responses (PID-a, -b and-c).
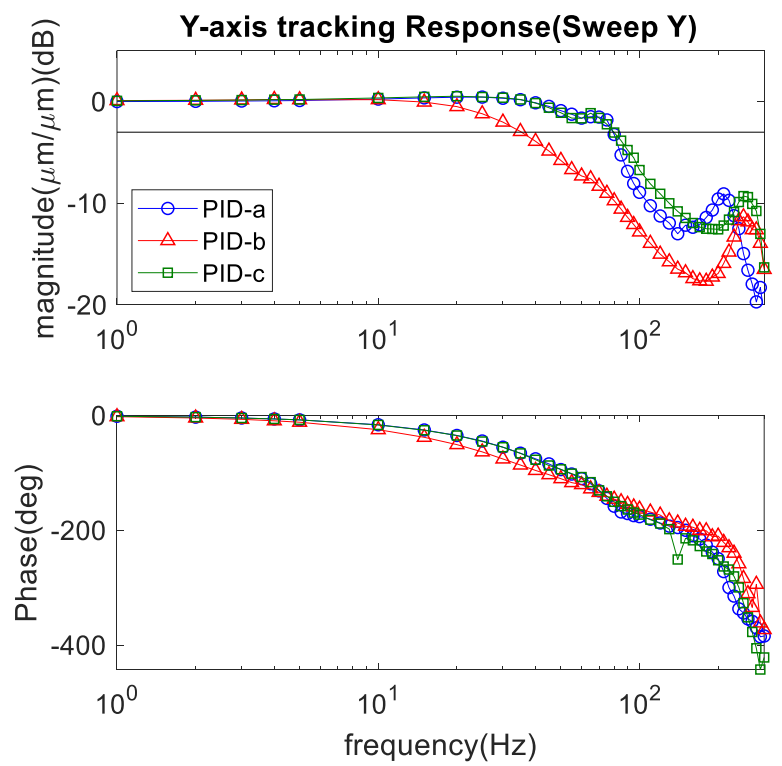

Fig. 12. Bode plot of the close-loop system (PID-a, -b and-c). 


\subsection{Comparison with different controller}

On the other hand, original plant with ISMC has been selected to compare with PID-a. ISMC with a parameter set of $\lambda=0.0018, \eta=50, \phi=0.005$ and $\beta=8$ reaches a rise time of $3.9 \mathrm{~ms}$ and settling time of $20.9 \mathrm{~ms}$, which is shown in Fig. 13. The sinusoidal test result is shown in Fig. 14, the $-3 \mathrm{~dB}$ bandwidth is $110 \mathrm{~Hz}$ by using ISMC. Table 3 summarizes the performance of the control result in these setups. In current works, the ISMC has better settling time and bandwidth in $Y$-axis. The $\theta_{x}$ and $\theta_{z}$ axis has steady state error because only one controller is applied in command axis( $Y$-axis). In future work, controllers of $\theta_{x}$ and $\theta_{z}$ axis will be designed and added in control system to decrease steady state errors which cause by coupled effect.

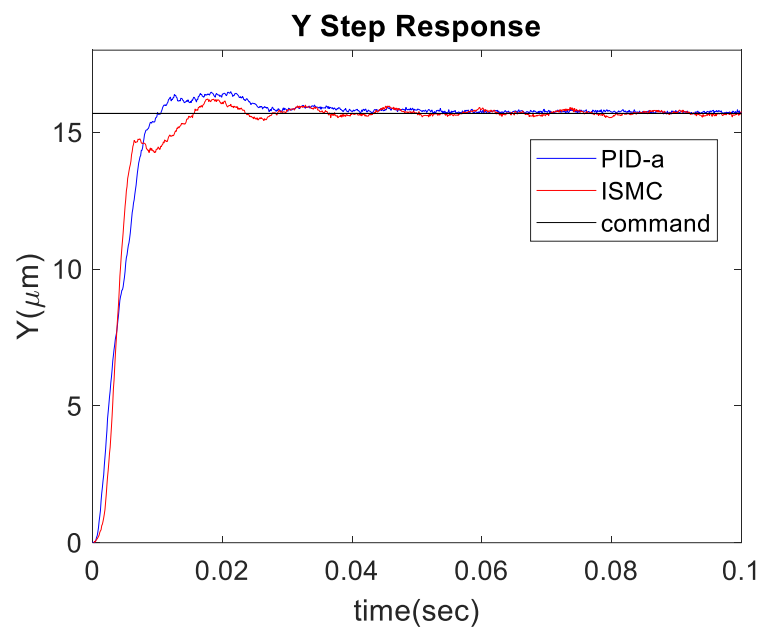

Fig. 13. Step responses (PID-a and ISMC).
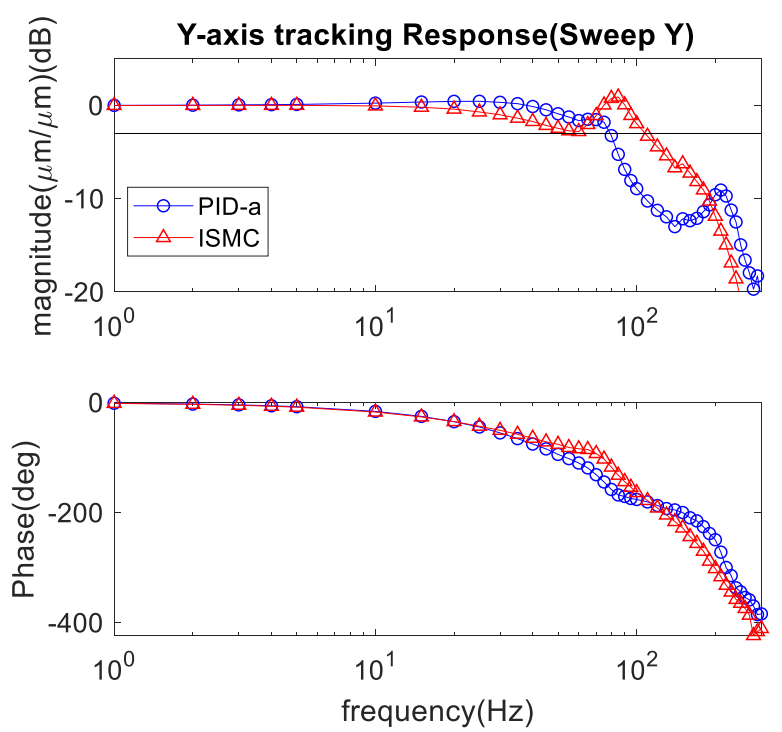

Fig. 14. Bode plot of the close-loop system (PID-a and ISMC).
Table 3. Performance of the system under control.

\begin{tabular}{|c|c|c|c|c|}
\hline & PID-a & PID-b & PID-c & ISMC \\
\hline Rise time (ms) & 6 & 11.8 & 5.8 & 3.9 \\
\hline Settling time (ms) & 25.3 & 41.4 & 24.3 & 20.9 \\
\hline Overshoot (\%) & 5.1 & 4.4 & 7.4 & 3.3 \\
\hline Bandwidth (Hz) & 79 & 35 & 79 & 110 \\
\hline
\end{tabular}

\section{Conclusions}

In this work, a stiffness-tunable 3-DOF rubber bearing positioning stage has been developed, the design and control of the stage is presented for dynamic characteristic adjustment and positioning control. The system is actuated by 3 voice coil motor. 3 capacitive probes are used to sense one translational and two rotational DOF. PID controller and ISMC are designed for performing the motion control. Currently, the stiffness-tunable design shows that the dynamic characteristics of this stage is adjustable. This is an advantage compare with compliant stage. For example, the length of compliant mechanism needs to be decreased by $30 \%$ to make the stiffness increase by $100 \%$, that makes the size of compliant stage have large adjustment. The stiffness of the rubber bearing stage can increase by $100 \%$ and by $510 \%$ in translational direction and in rotational direction with small scale adjustment, which is more efficiently than compliant stage. The $-3 \mathrm{~dB}$ bandwidth of controlling $Y$-axis is $79 \mathrm{~Hz}$ by PID control. With ISMC, the bandwidth can reach $110 \mathrm{~Hz}$. In current work the stage with rubber adjustment can approaching same control result to original stage. The future work of this research is to finish controller design of all three axes to realize coupling control. $\theta_{x}$ and $\theta_{z}$ -axis control experiment with preload will get on. It could improve control performance with $\theta_{x}$ and $\theta_{z}$-axis control testing. With further future, this rubber bearing stage should be useful for various applications such as precision engineering or laser scanning.

\section{Acknowledgment}

This work is supported by Ministry of Science and Technology (MOST) of Taiwan under contract numbers: MOST 105-2221-E-006-100-MY3, 108-2221-E-006-209, 108-2622-8-006-014, and 108-2221-E-218-027. Support from Y-C Teng of NCKU during the early stages of this work are greatly appreciated. 


\section{References}

(1) K. S. Chen, D.L. Trumper, S.T. Smith, "Design and control for an electromagnetically driven $\mathrm{X}-\mathrm{Y}-\theta$ stage," Journal of the International Societies for Precision Engineering and Nanotechnology 26 (2002) 355-369.

(2) G. C. Loney, "High bandwidth steering mirror research", Project Report IRP-15, MIT Lincoln Laboratories, Lexington, MA, January 1992.

(3) W. C. Wang, J. W. Lee, K. S. Chen, Y. H. Liu, "Design and vibration control of a notch-based compliant stage for display panel inspection applications", Journal of Sound and Vibration, Vol. 333, No. 10, pp.2701-2718, 2014.

(4) H. C. Tsai and J. Kelly, "Bending stiffness of fiber-reinforced circular seismic isolators", Journal of Engineering Mechanics, Vol. 128, pp. 1150-1157, (2002).

(5) A. N. Gent, "Engineering with Rubber : how to design rubber components $3 \mathrm{rd}$ ed", Hanser Publications, Cincinnati, 2002.

(6) D. Kluk, M. Boulet, D. L. Trumper, "A high-bandwidth, high-precision, two-axis steering mirror with moving iron actuator", IFAC Proceedings Volumes, Vol. 43, No. 18, pp.552-557, 2010.

(7) Y. C. Teng and K. S. Chen, "Analysis, Design, and Control of a Novel Elastomeric Bearing Positioning Stage", Inventions 2016, Vol. 1, No. 3, 2016.

(8) Y. C. Chen, "Design, Analysis and Control of a Novel Triaxial Elastomeric Bearing Positioning Stage", Master Thesis, National Cheng-Kung University, Taiwan, 2017.

(9) R. Christensen, "Theory of viscoelasticity: an introduction", Elsevier, 2012.

(10) N. S. Nise, “Control Systems Engineering”, John Wiley \& Sons, 2007

(11) J. J. E. Slotine and W. Li, "Applied nonlinear control", Prentice-Hall, 1991. 